Network Working Group

Request for Comments: 2591

Category: Standards Track
D. Levi

Nortel Networks

J. Schoenwaelder

TU Braunschweig

May 1999

\title{
Definitions of Managed Objects for \\ Scheduling Management Operations
}

\section{Status of this Memo}

This document specifies an Internet standards track protocol for the Internet community, and requests discussion and suggestions for improvements. Please refer to the current edition of the "Internet Official Protocol Standards" (STD 1) for the standardization state and status of this protocol. Distribution of this memo is unlimited.

Copyright Notice

Copyright (C) The Internet Society (1999). All Rights Reserved.

Abstract

This memo defines a portion of the Management Information Base (MIB) for use with network management protocols in the Internet community. In particular, it describes a set of managed objects that are used to schedule management operations periodically or at specified dates and times.

Table of Contents

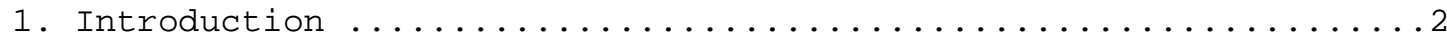

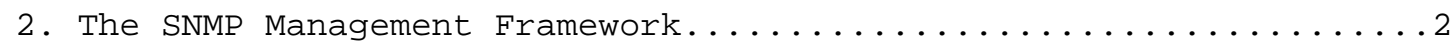

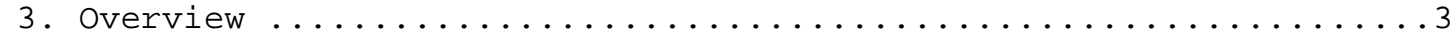

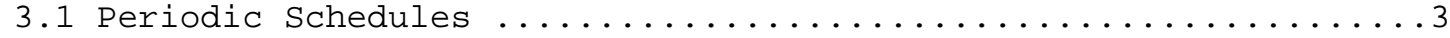

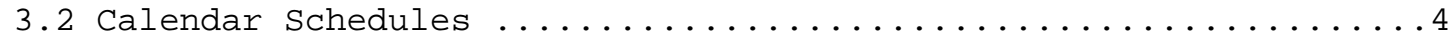

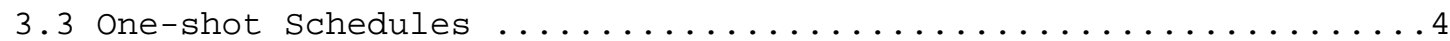

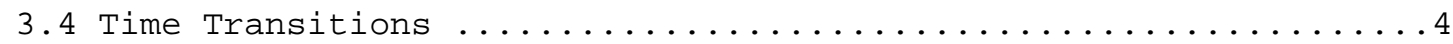

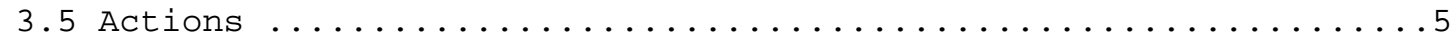

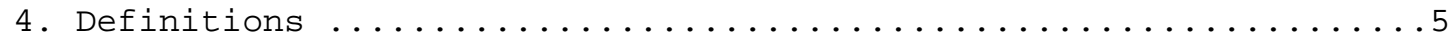

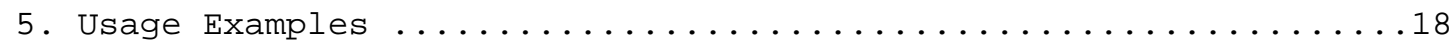

5.1 Starting a script to ping devices every 20 minutes .........18

5.2 Starting a script at the next Friday the 13 th $\ldots \ldots \ldots \ldots \ldots$

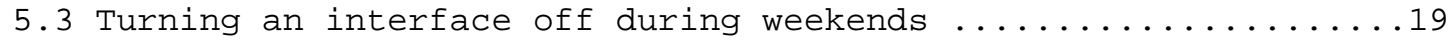

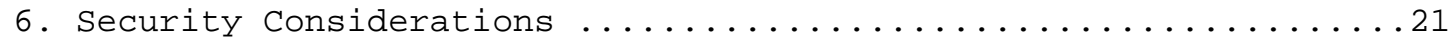

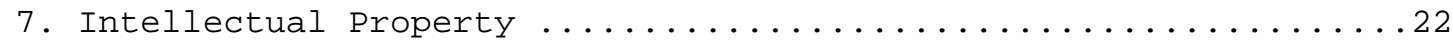

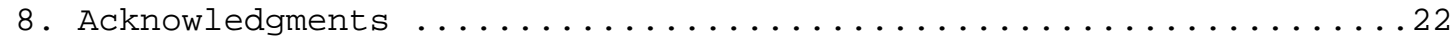




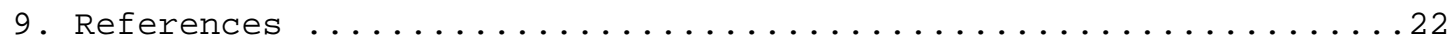

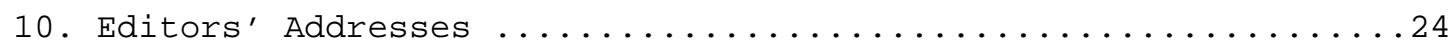

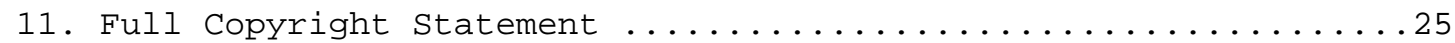

\section{Introduction}

This memo defines a portion of the Management Information Base (MIB) for use with network management protocols in the Internet community. In particular, it describes a set of managed objects that are used to schedule management operations periodically or at specified dates and times.

The key words "MUST", "MUST NOT", "REQUIRED", "SHALL", "SHALL NOT", "SHOULD", "SHOULD NOT", "RECOMMENDED", "MAY", and "OPTIONAL" in this document are to be interpreted as described in RFC 2119 [19].

\section{The SNMP Management Framework}

The SNMP Management Framework presently consists of five major components:

- An overall architecture, described in RFC 2271 [1].

- Mechanisms for describing and naming objects and events for the purpose of management. The first version of this structure of Management Information (SMI) is called SMIV1 and described in STD 16, RFC 1155 [2], STD 16, RFC 1212 [3] and RFC 1215 [4]. The second version, called SMIV2, is described in STD 58, RFC 2578 [5], RFC 2579 [6] and RFC 2580 [7].

- Message protocols for transferring management information. The first version of the SNMP message protocol is called SNMPV1 and described in RFC 1157 [8]. A second version of the SNMP message protocol, which is not an Internet standards track protocol, is called SNMPv2c and described in RFC 1901 [9] and RFC 1906 [10]. The third version of the message protocol is called SNMPv3 and described in RFC 1906 [10], RFC 2272 [11] and RFC 2274 [12].

- Protocol operations for accessing management information. The first set of protocol operations and associated PDU formats is described in STD 15, RFC 1157 [8]. A second set of protocol operations and associated PDU formats is described in RFC 1905 [13].

- A set of fundamental applications described in RFC 2273 [14] and the view-based access control mechanism described in RFC 2275 [15]. 
Managed objects are accessed via a virtual information store, termed the Management Information Base or MIB. Objects in the MIB are defined using the mechanisms defined in the SMI.

This memo specifies a MIB module that is compliant to the SMIV2. A MIB conforming to the SMIv1 can be produced through the appropriate translations. The resulting translated MIB must be semantically equivalent, except where objects or events are omitted because no translation is possible (use of Counter64). Some machine readable information in SMIV2 will be converted into textual descriptions in SMIv1 during the translation process. However, this loss of machine readable information is not considered to change the semantics of the MIB.

3. Overview

The MIB defined in this memo provides scheduling of actions periodically or at specified dates and times. The actions can be used to realize on-duty / off-duty schedules or to trigger management functions in a distributed management application.

Schedules can be enabled or disabled by modifying a control object. This allows pre-configured schedules which are activated or deactivated by some other management functions.

The term 'scheduler' is used throughout this memo to refer to the entity which implements the scheduling MIB and which invokes the actions at the specified points in time.

\subsection{Periodic Schedules}

Periodic schedules are based on fixed time periods between the initiation of scheduled actions. Periodic schedules are defined by specifying the number of seconds between two initiations. The time needed to complete the action is usually not known by the scheduler and does therefore not influence the next scheduling point.

Implementations must guarantee that action invocations will not occur before their next scheduled time. However, implementations may be forced to delay invocations in the face of local constraints (e.g., a heavy load on higher-priority tasks). An accumulation of such delays would result in a drift of the scheduling interval with respect to time, and should be avoided.

Scheduled actions collecting statistical data should retrieve time stamps from the data source and not rely on the accuracy of the periodic scheduler in order to obtain accurate statistics. 


\subsection{Calendar Schedules}

Calendar schedules trigger scheduled actions at specified days of the week and days of the month. Calendar schedules are therefore aware of the notion of months, days, weekdays, hours and minutes.

It is possible to specify multiple values for each calendar item. This provides a mechanism for defining complex schedules. For example, a schedule could be defined which triggers an action every 15 minutes on a given weekday.

Months, days and weekdays are specified using the objects schedMonth, schedDay and schedWeekDay of type BITS. Setting multiple bits to one in these objects causes an OR operation. For example, setting the bits monday(1) and friday(5) in schedWeekDay restricts the schedule to Mondays and Fridays.

The bit fields for schedMonth, schedDay and schedWeekDay are combined using an AND operation. For example, setting the bits june (5) and july(6) in schedMonth and combining it with the bits monday(1) and friday(5) set in schedWeekDay will result in a schedule which is restricted to every Monday and Friday in the months June and July. Wildcarding of calendar items is achieved by setting all bits to one.

It is possible to define calendar schedules that will never trigger an action. For example, one can define a calendar schedule which should trigger an action on February 31st. Schedules like this will simply be ignored by the scheduler.

Finally, calendar schedules are always expressed in local time. A scalar, schedLocaltime is provided so that a manager can retrieve the notion of local time and the offset to GMT time.

\subsection{One-shot Schedules}

One-shot Schedules are similar to calendar schedules. The difference between a calendar schedule and a one-shot schedule is that a oneshot schedule will automatically disable itself once an action has been invoked.

\subsection{Time Transitions}

When a system's notion of time is changed for some reason, implementations of the schedule MIB must schedule actions differently. One example of a change to a system's notion of time is when a daylight savings time transition occurs. 
There are two possible situations when a time transition occurs. First, time may be set backwards, in which case particular times will appear to occur twice within the same day. These are called 'ambiguous times'. Second, time may be set forwards, in which case particular times will appear to not occur within a day. This are called 'nonexistent times'.

When an action is configured in the Schedule MIB to occur at an ambiguous time during a time transition, the action SHALL only be invoked at the first occurence of the ambiguous time. For example, if an action is scheduled to occur at 2:00 am, and a time transition occurs at 3:00 am which sets the clock back to 2:00 am, the action SHALL only be invoked at the first occurence of 2:00 am.

When an action is configured in the Schedule MIB to occur at a nonexistent time, the action SHOULD be invoked immediately upon a time transition. If multiple actions are invoked in this way, they SHALL be invoked in the order in which they normally would be invoked had the time transition not occured. For example, if an action (a) is scheduled at 2:05 am and another action (b) at 2:10 am, then both actions SHOULD be invoked at 3:00 am in the order (a), (b) if the time jumps forward from 2:00 am to 3:00 am.

\subsection{Actions}

Scheduled actions are modeled by SNMP set operations on local MIB variables. Scheduled actions described in this MIB are further restricted to objects of type INTEGER. This restriction does not limit the usefulness of the MIB. Simple schedules such as on-duty / off-duty schedules for resources that have a status MIB object (e.g. ifAdminstatus) are possible.

More complex actions can be realized by triggering a management script which is responsible for performing complex state transitions. A management script can also be used to perform SNMP set operations on remote SNMP engines.

4. Definitions

DISMAN-SCHEDULE-MIB DEFINITIONS : := BEGIN

IMPORTS

MODULE-IDENTITY, OBJECT-TYPE, NOTIFICATION-TYPE,

Integer32, Unsigned32, Counter32, mib-2 FROM SNMPV2-SMI

TEXTUAL-CONVENTION, DateAndTime, RowStatus, storageType, Variablepointer 


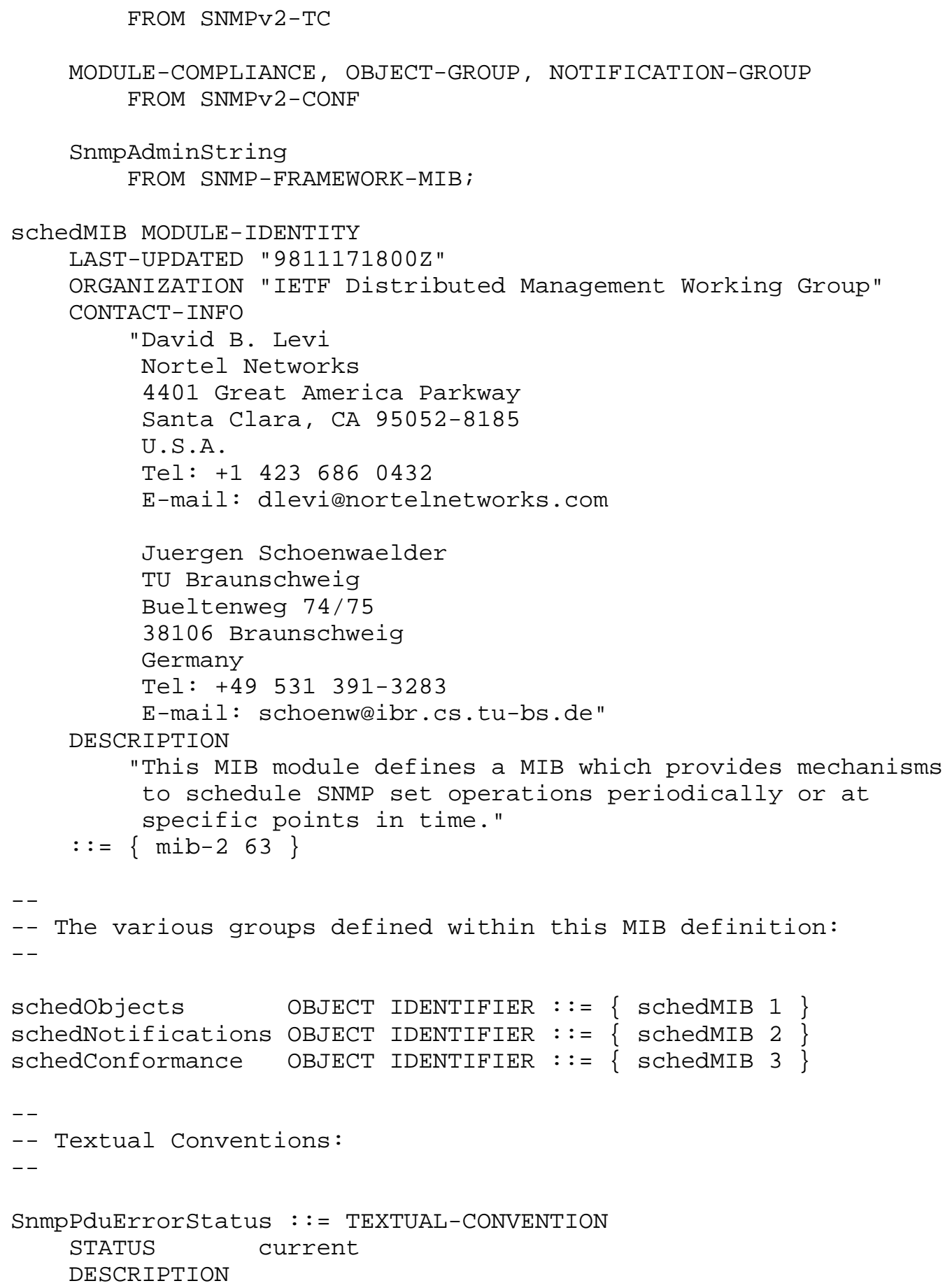




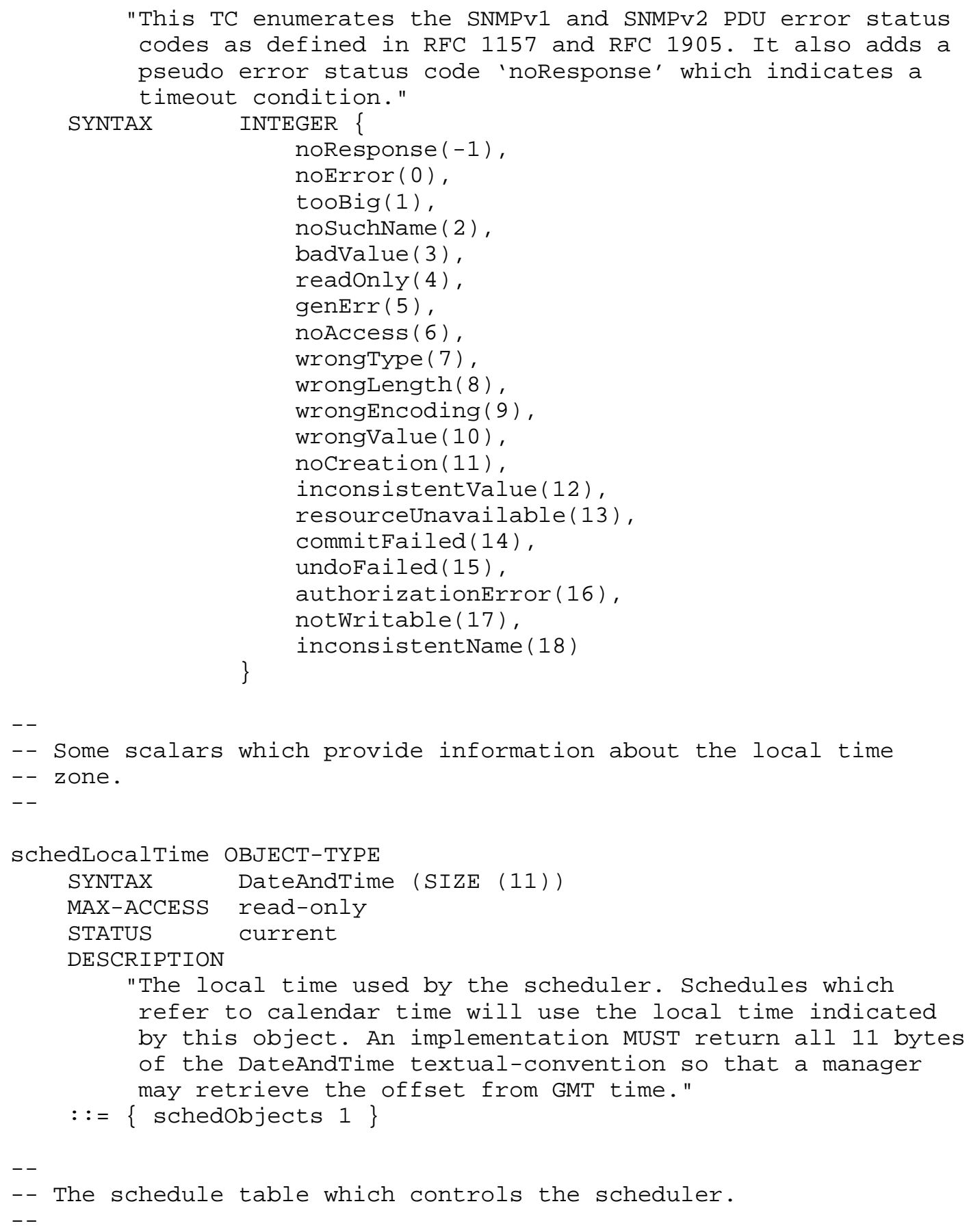




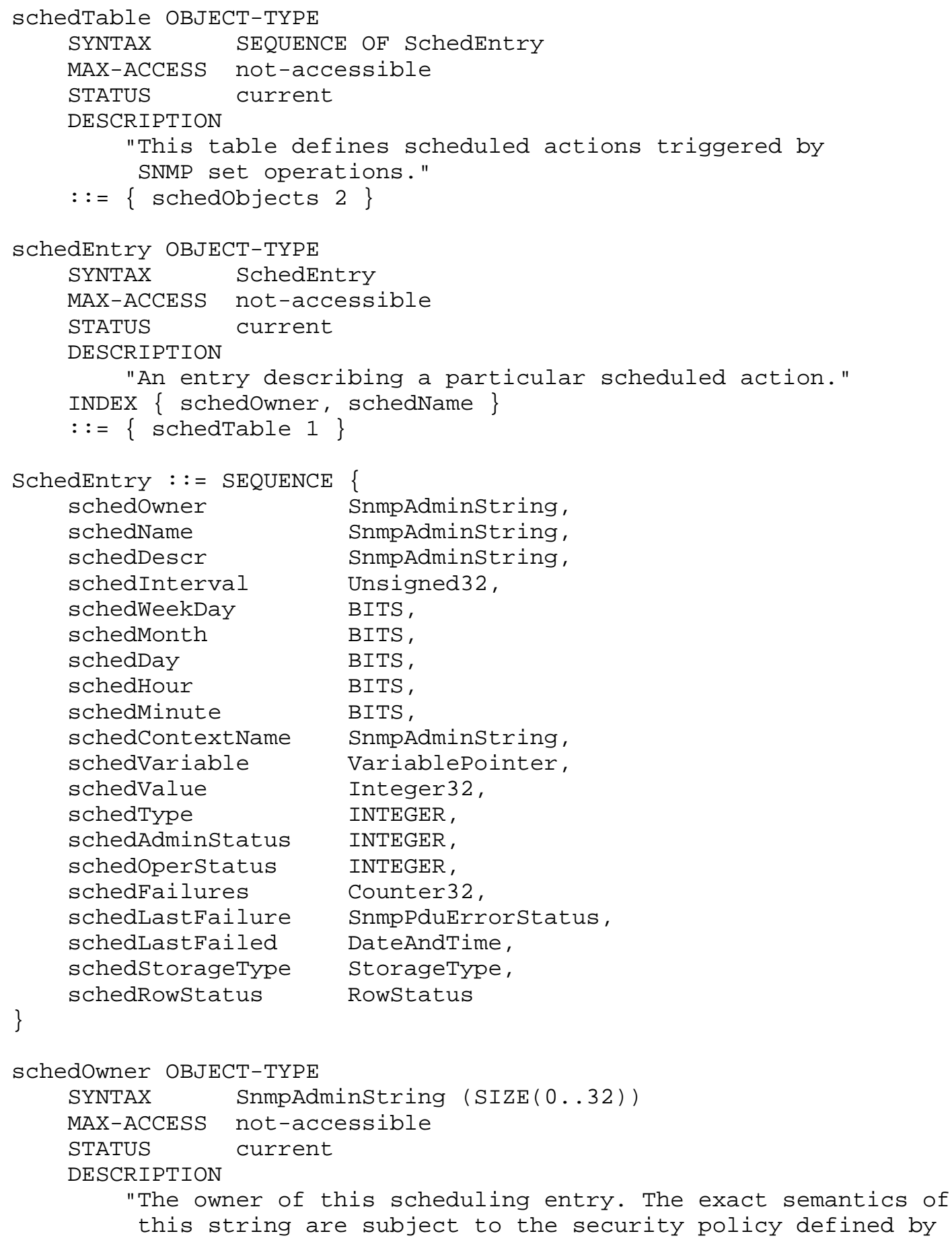




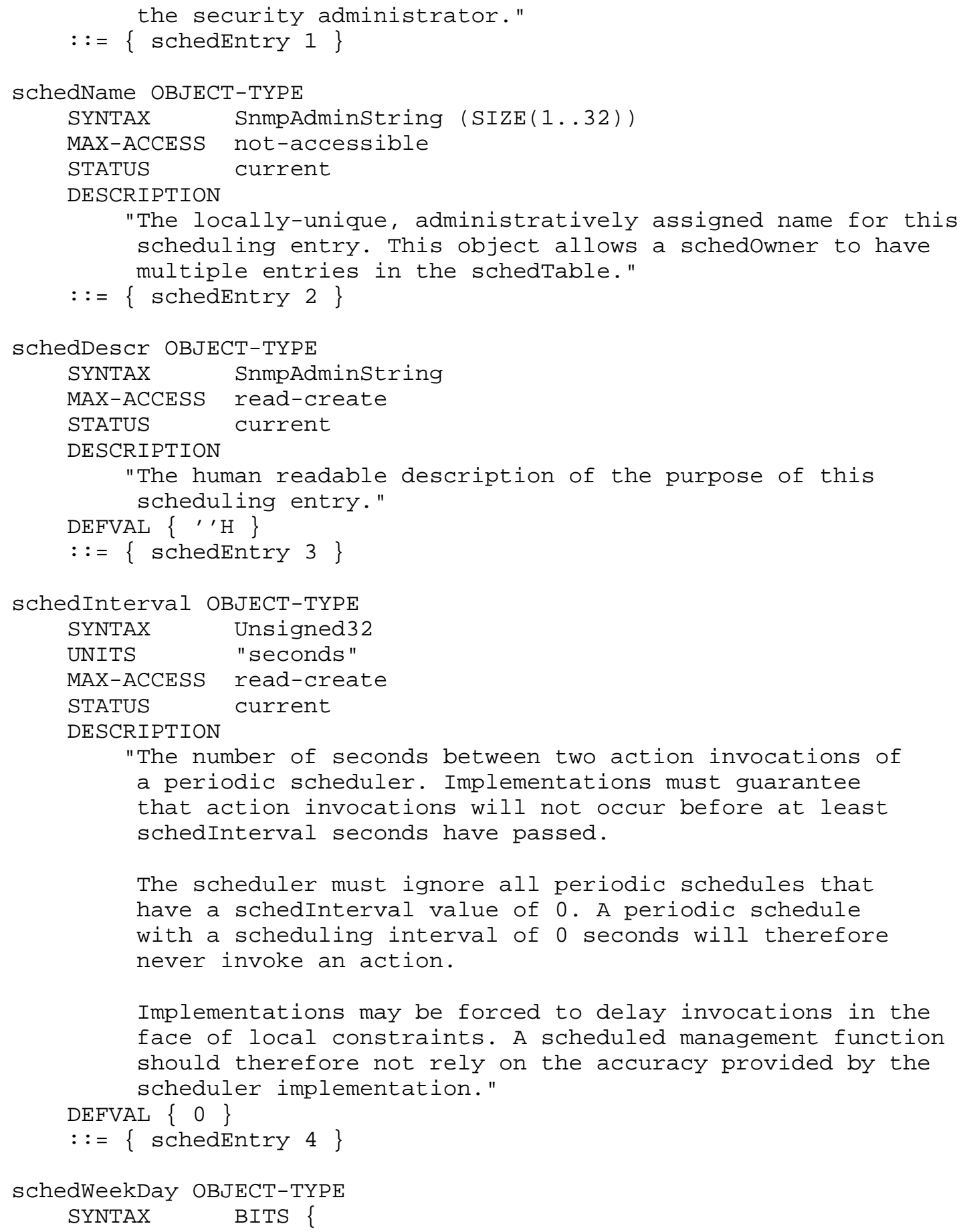




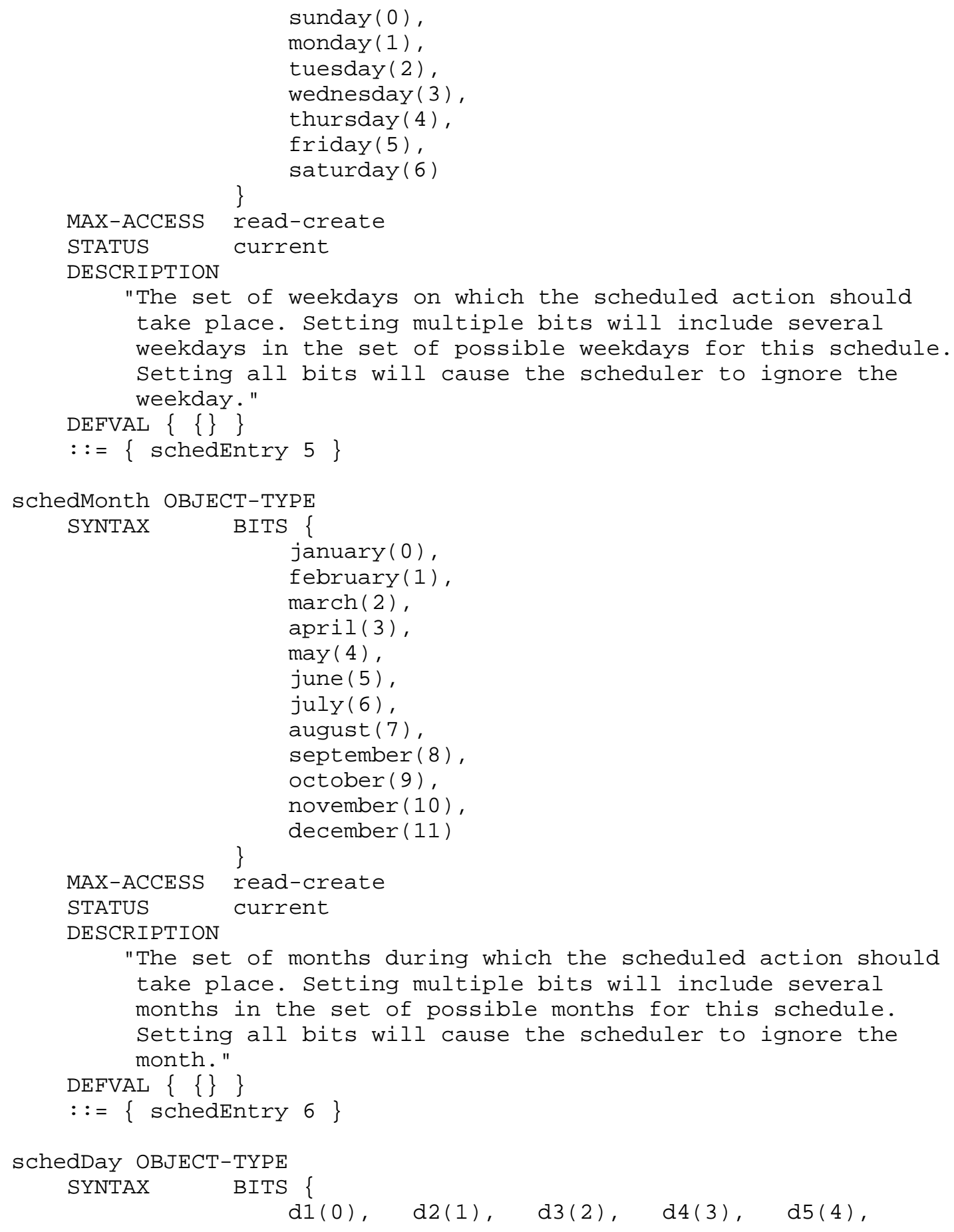




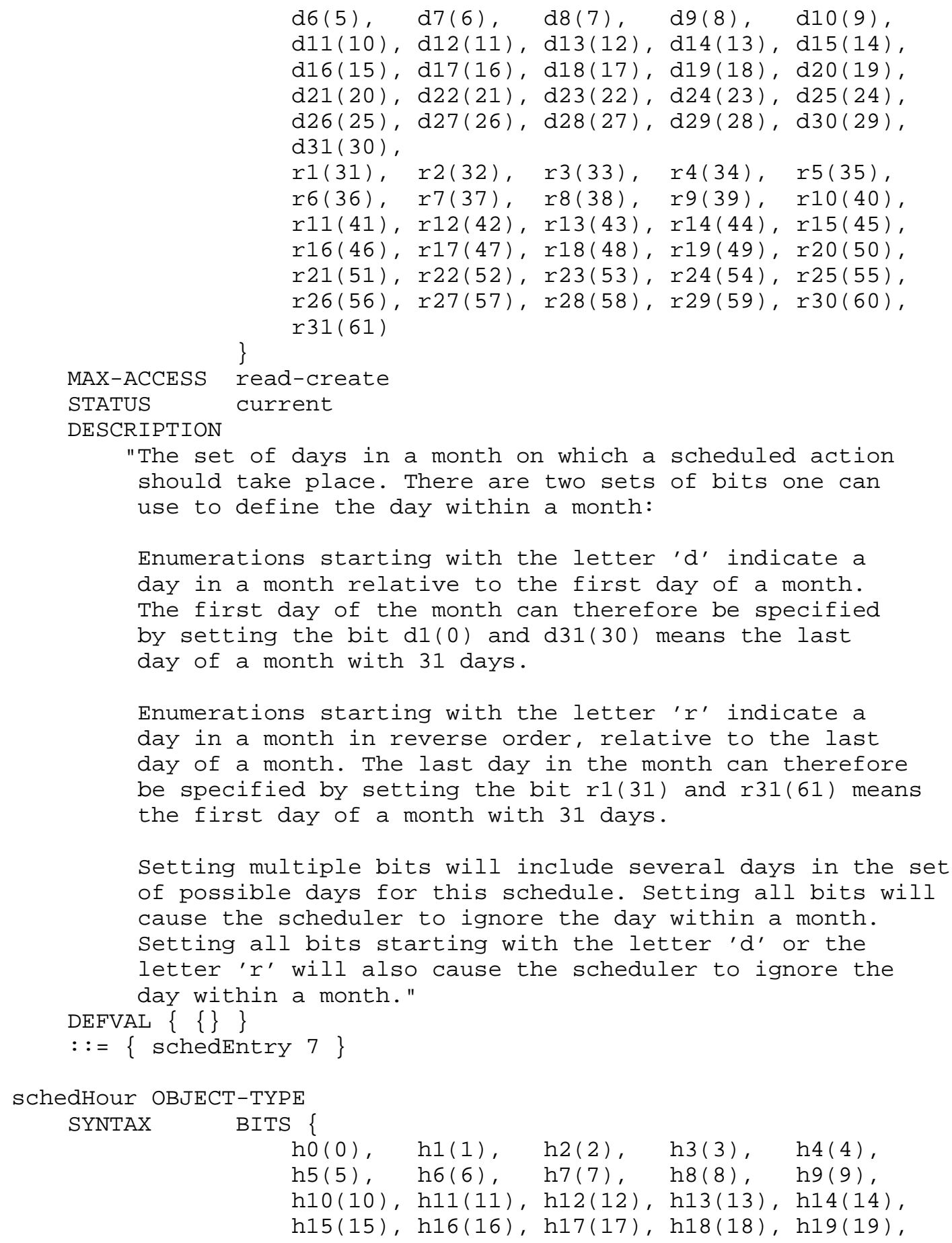




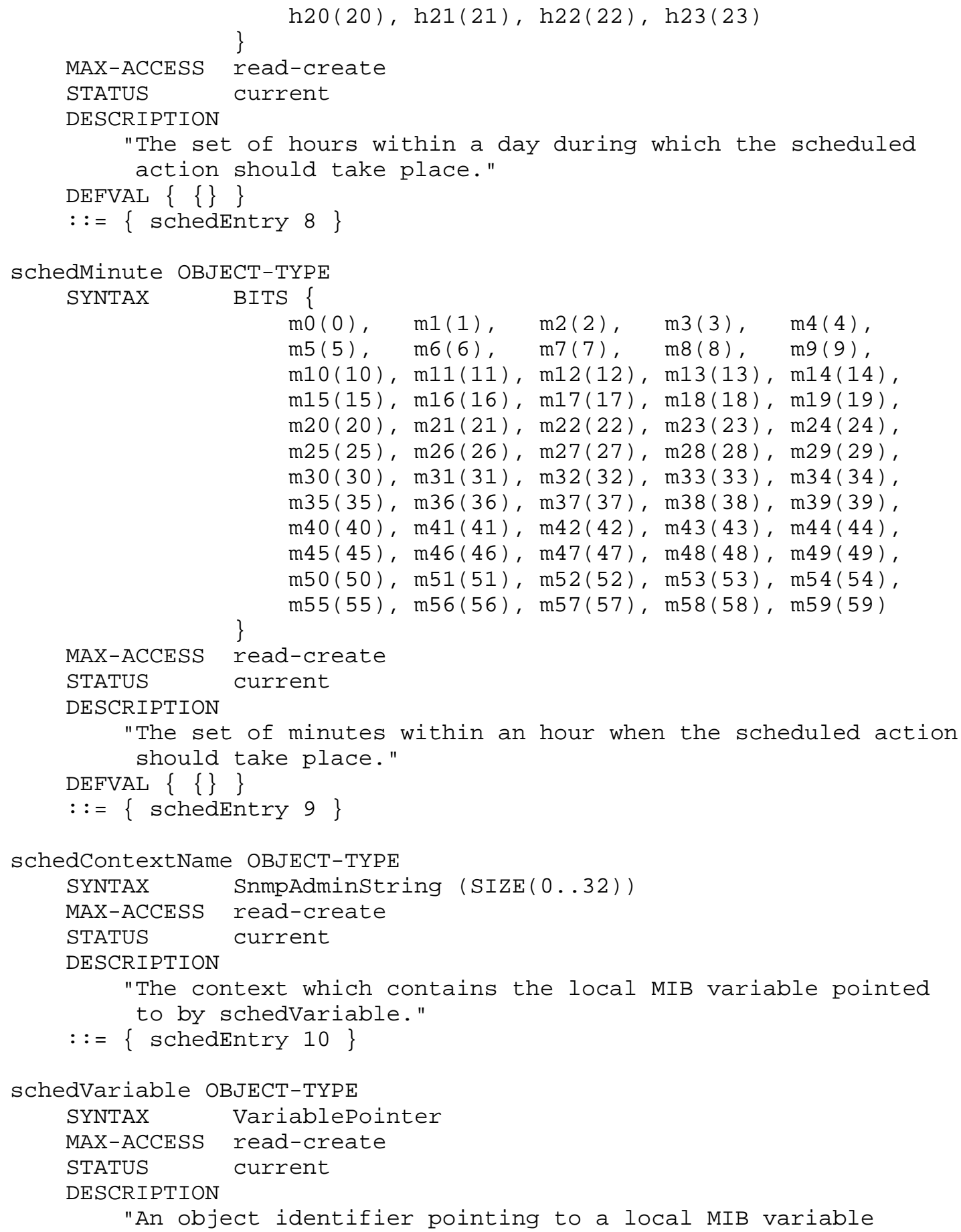




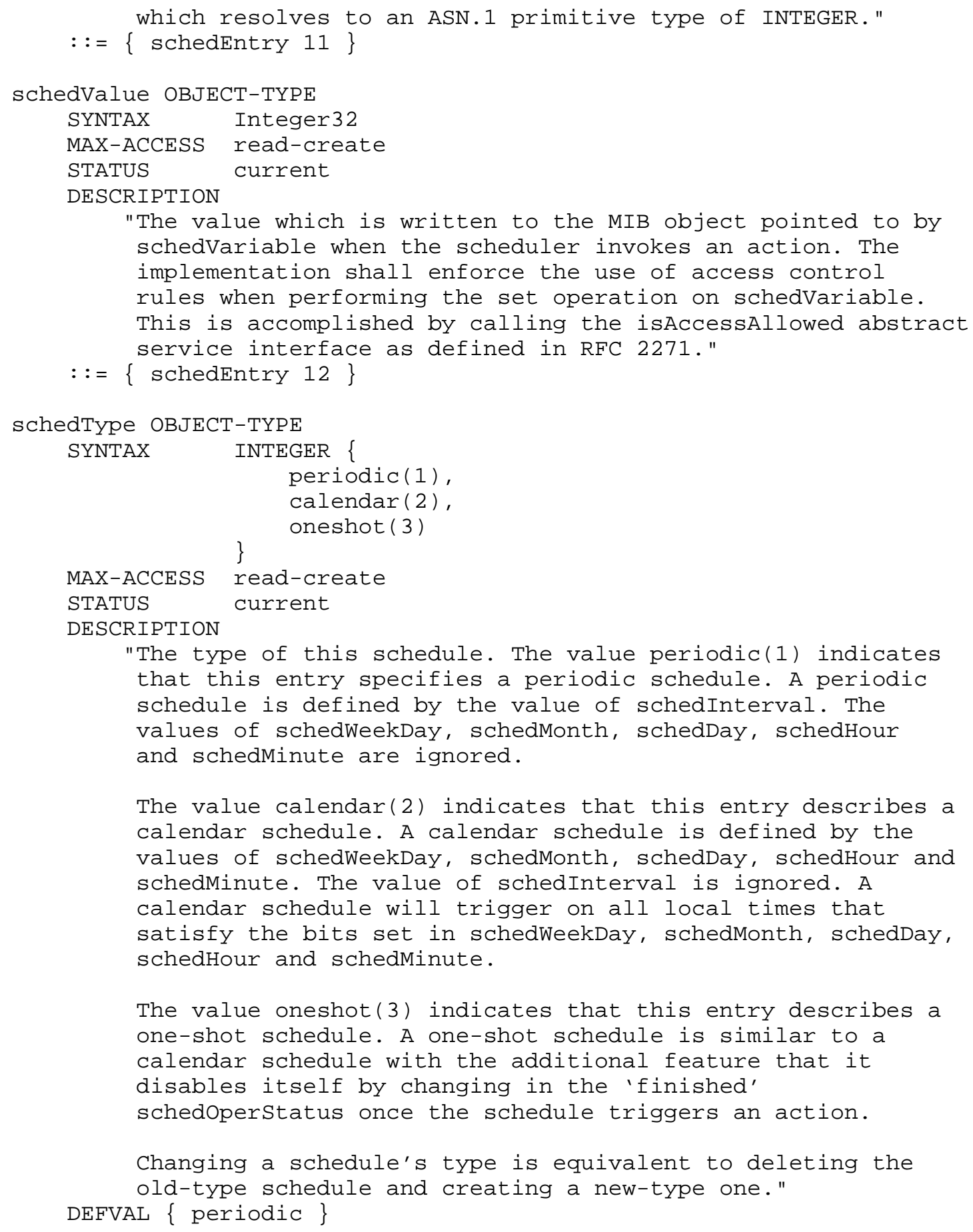




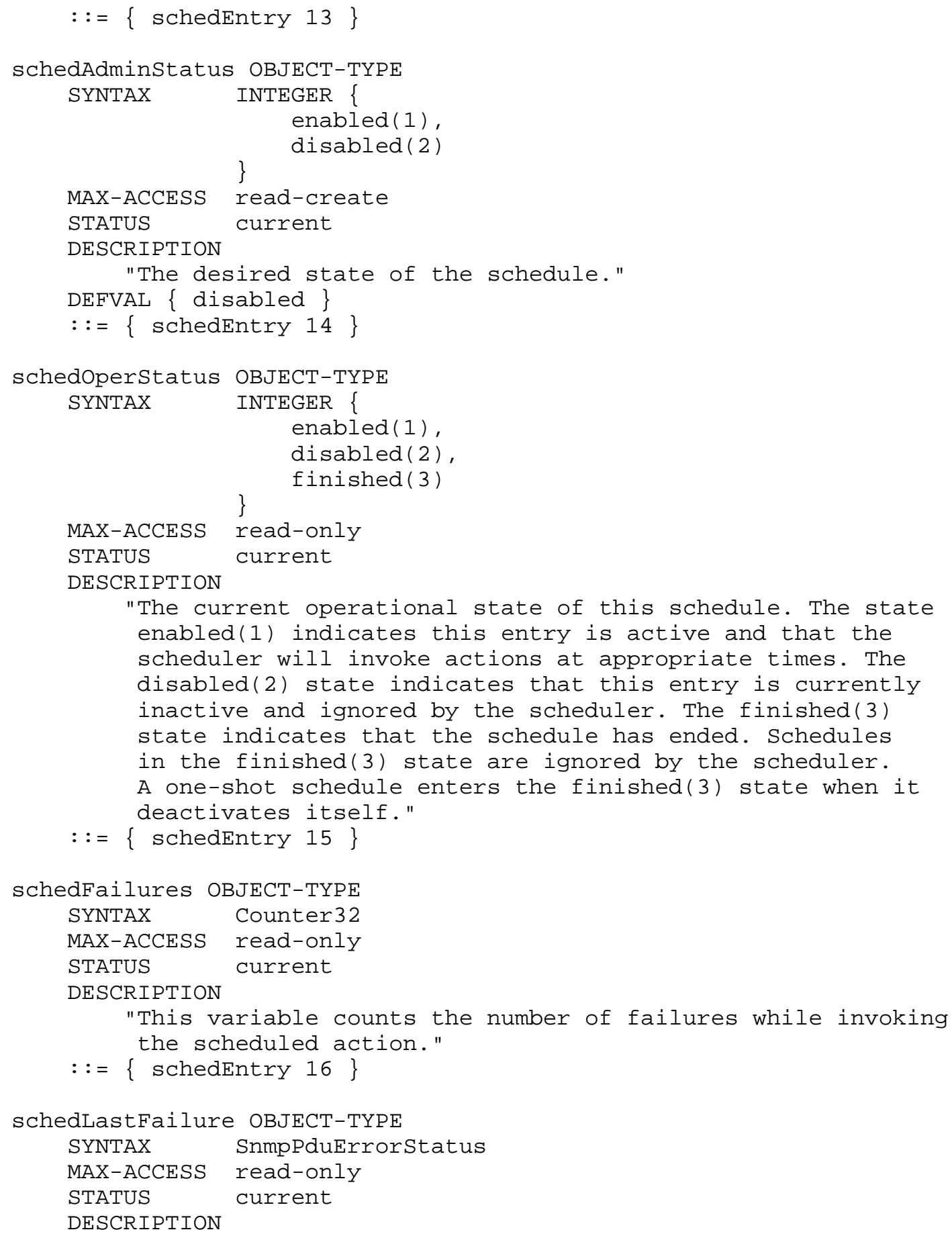




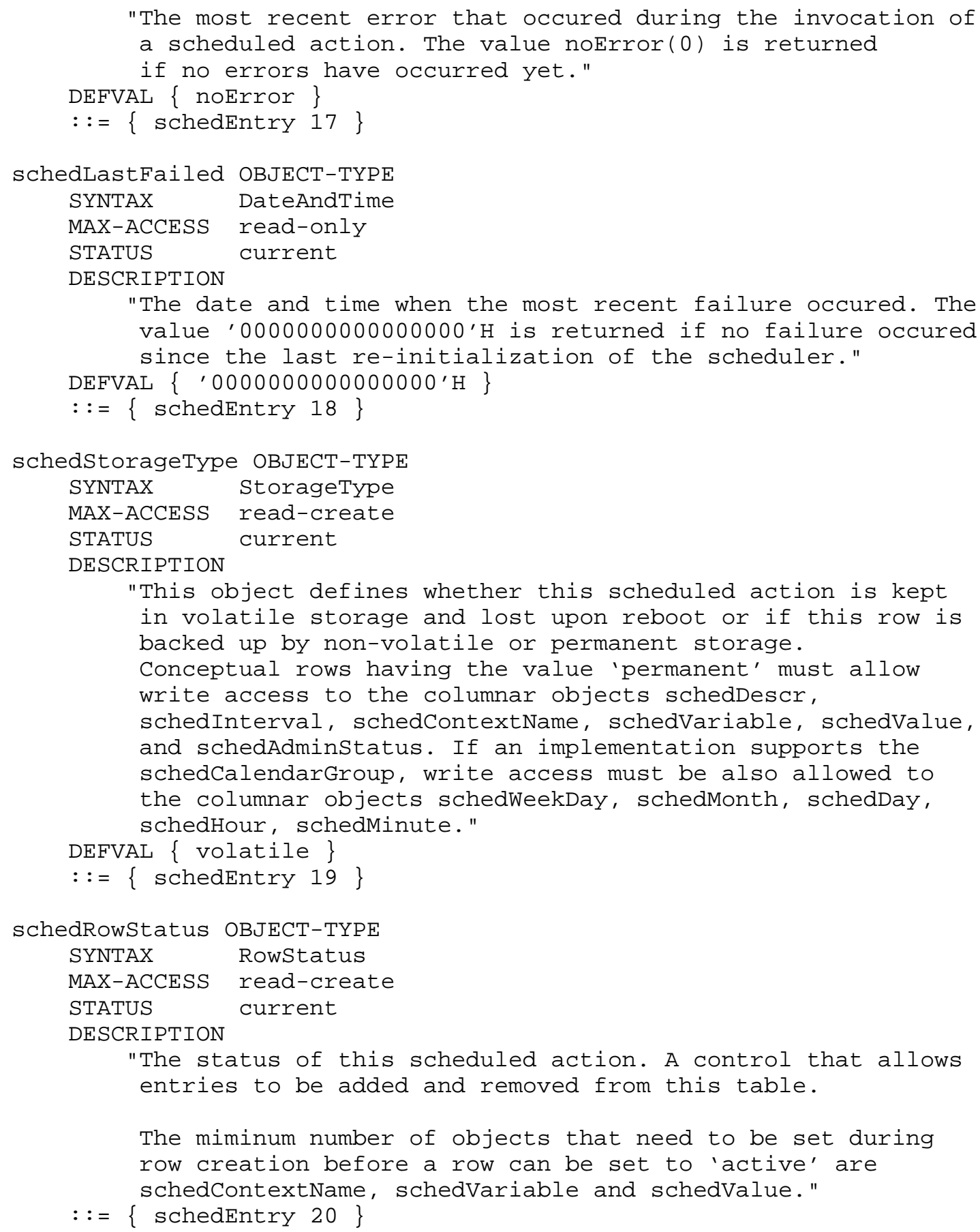




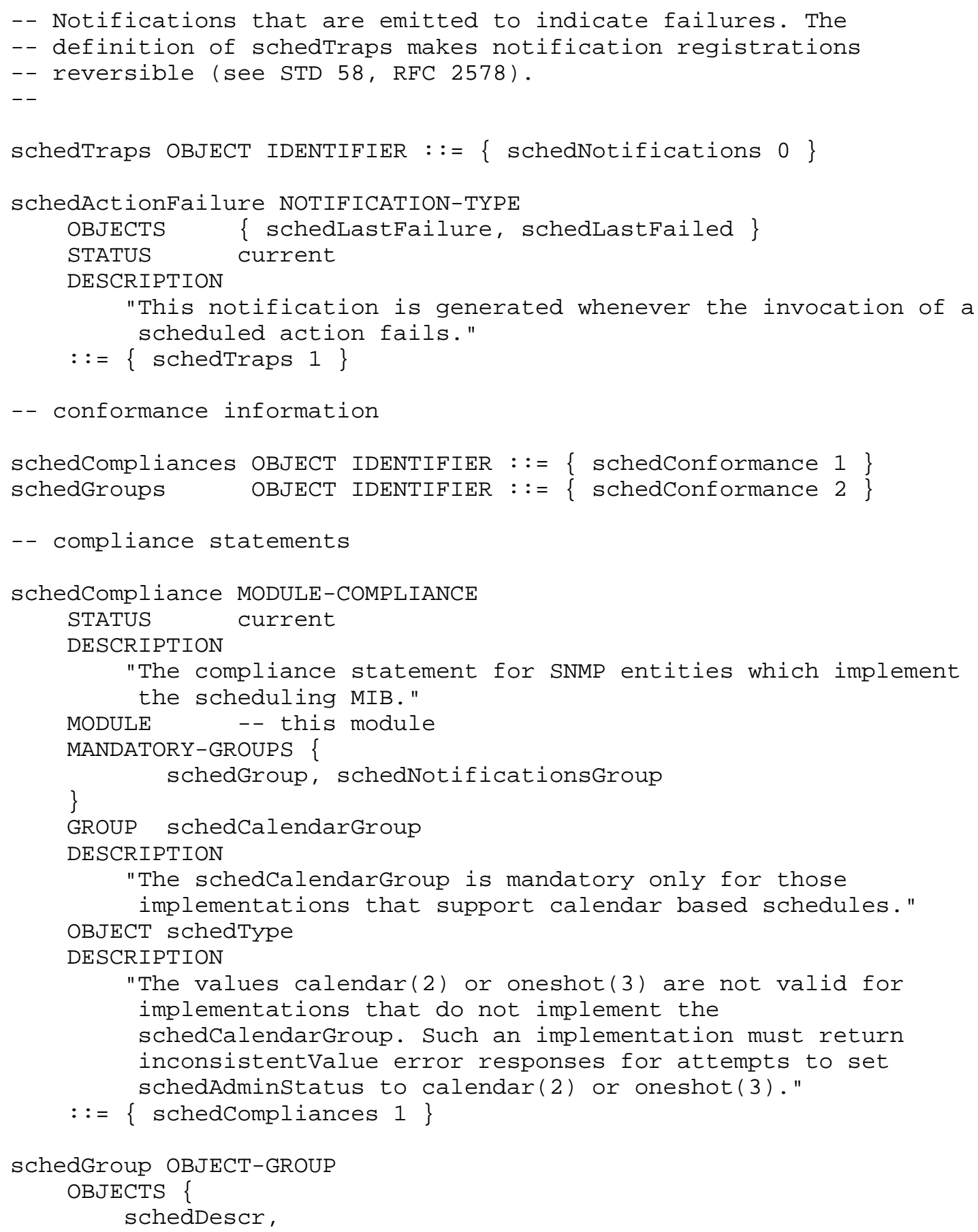




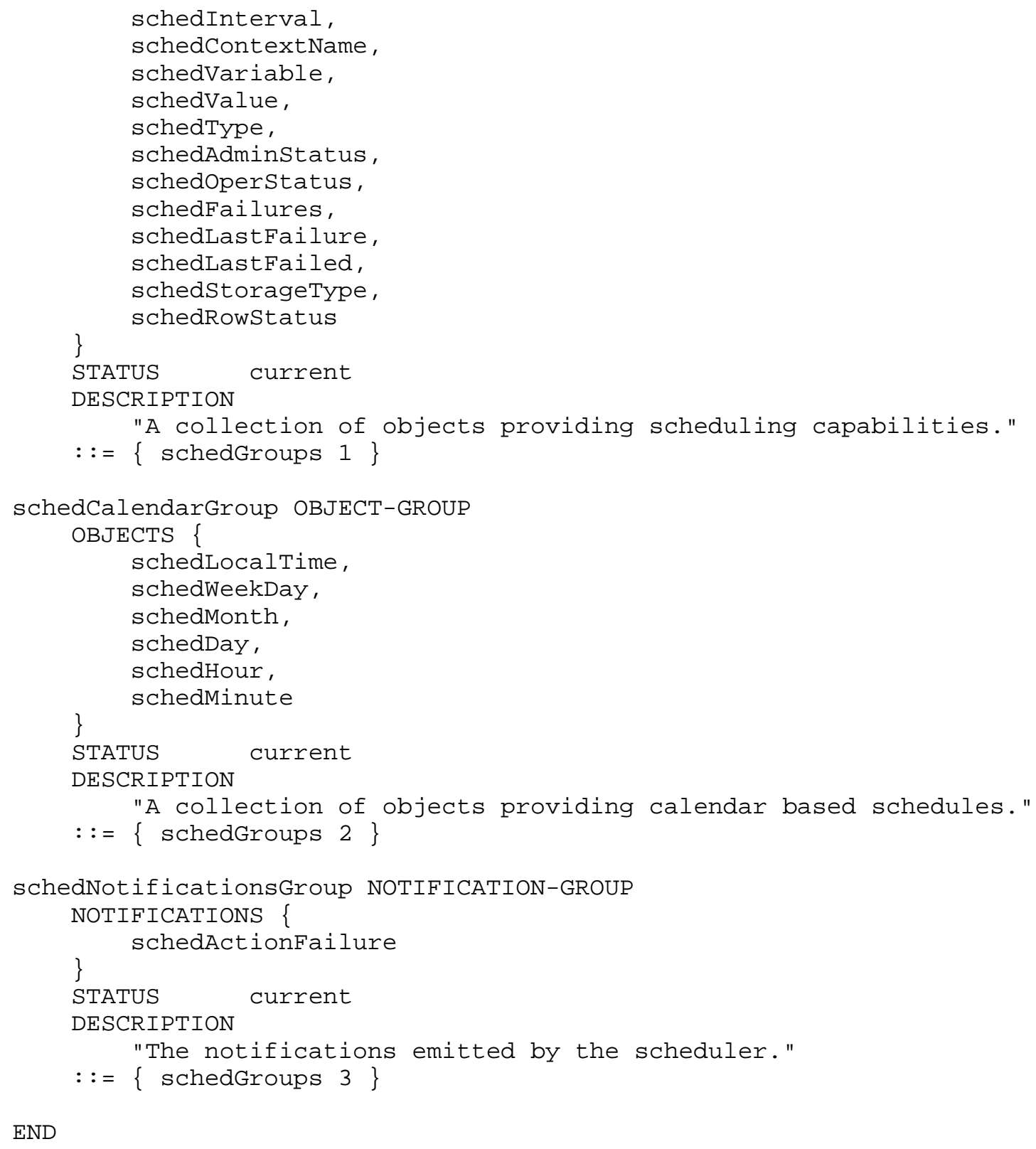

END 


\section{Usage Examples}

This section presents some examples how the scheduling MIB can be used to schedule scripts with the Script MIB [17] or to realize onduty/off-duty schedules by modifying status objects of other MIB modules.

5.1. Starting a script to ping devices every 20 minutes

It is assumed that the schedule entry is owned by schedOwner = "joe" and its name is schedName = "ping". The instance identifier for the scheduling entry is therefore 3.106.111.101.4.112.105.110.103.

It is further assumed that the smLaunchTable entry is owned by smLaunchowner $=$ "joe" and its name is smLaunchName = "ping-devs". The complete object identifier for the smLaunchstart object is therefore smLaunchStart.3.106.111.101.9.112.105.110.103.45.100.101.118.115. The script lives in the context identified by the string "engine1".

The configuration of the scheduler entry which launches the script every 20 minutes would look as follows:

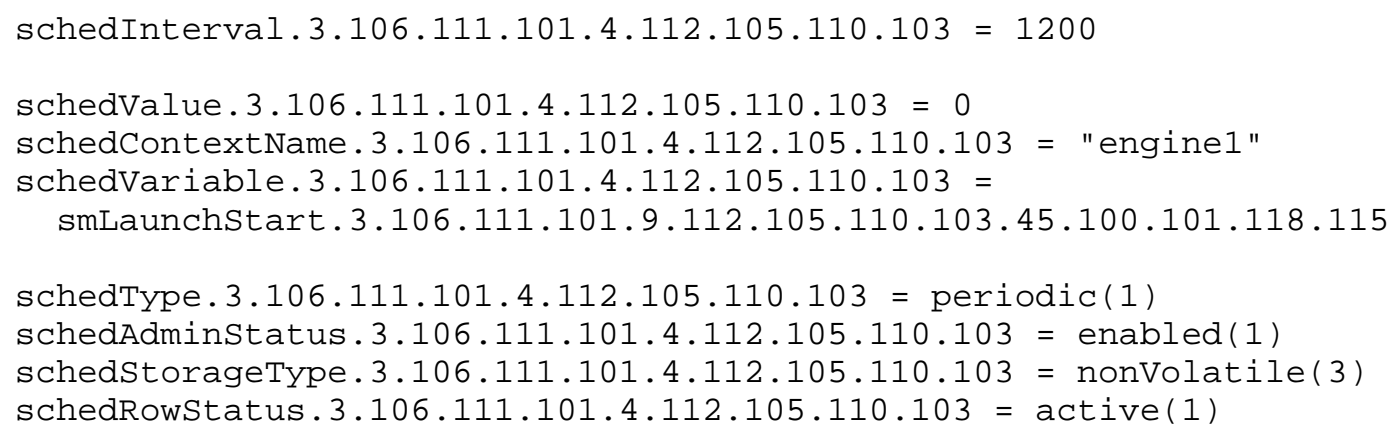

All the remaining columns in the schedTable represent status information and are not shown here.

5.2. Starting a script at the next Friday the 13th

It is assumed that the schedule entry is owned by schedOwner = "joe" and its name is schedName $=" 13 t h "$. The instance identifier for the scheduling entry is therefore 3.106.111.101.4.49.51.116.104.

It is further assumed that the smLaunchTable entry is owned by smLaunchowner = "joe" and its name is smLaunchName = "ghost". The complete object identifier for the smLaunchstart object is therefore smLaunchstart.3.106.111.101.5.103.104.111.115.116. The script lives in the context identified by the string "enginel". 
The configuration of the scheduler entry which launches the script on every Friday 13th at midnight would look as follows:

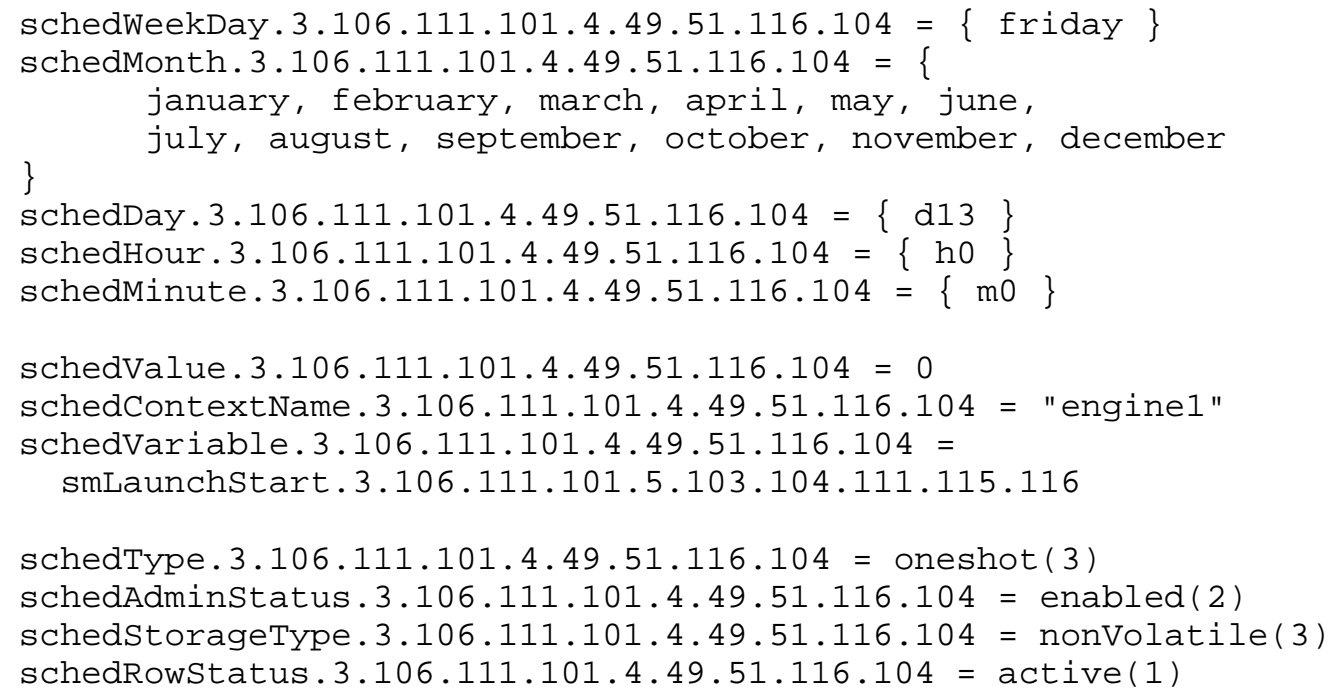

All the remaining columns in the schedTable represent status information and are not shown here.

\subsection{Turning an interface off during weekends}

This example assumes that a network interface should be taken down during weekends. The interface table (ifTable) of the IF-MIB [18] is assumed to exist in the context identified by an empty string and the index of the interface is ifIndex $=6$.

The scheduling entry which brings the interface down on every Friday evening at 20:30 (8:30 pm) is owned by schedOwner = "bob" and its name is schedName = "if-off". The instance identifier for the scheduling entry is therefore 3.98.111.98.6.105.102.45.111.102.102.

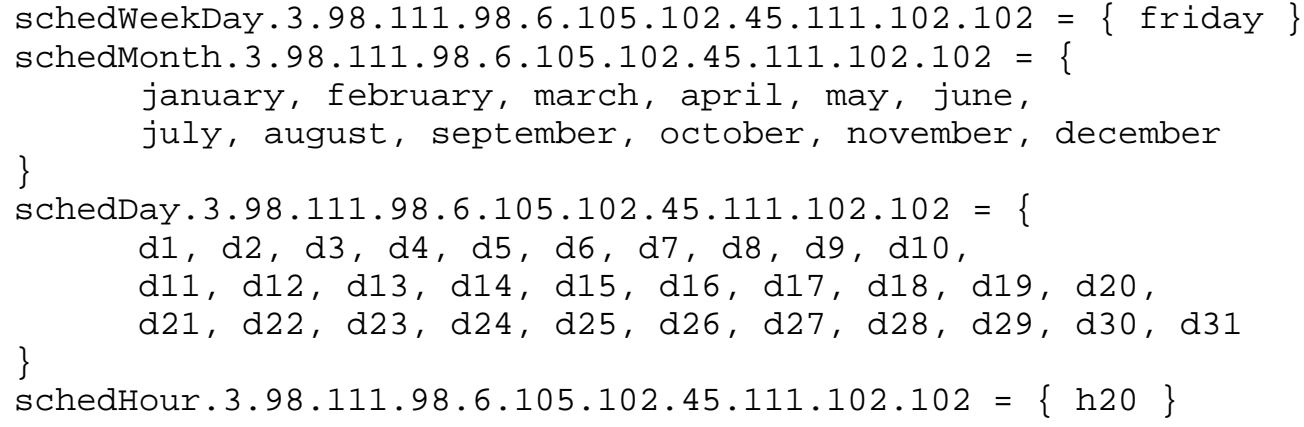




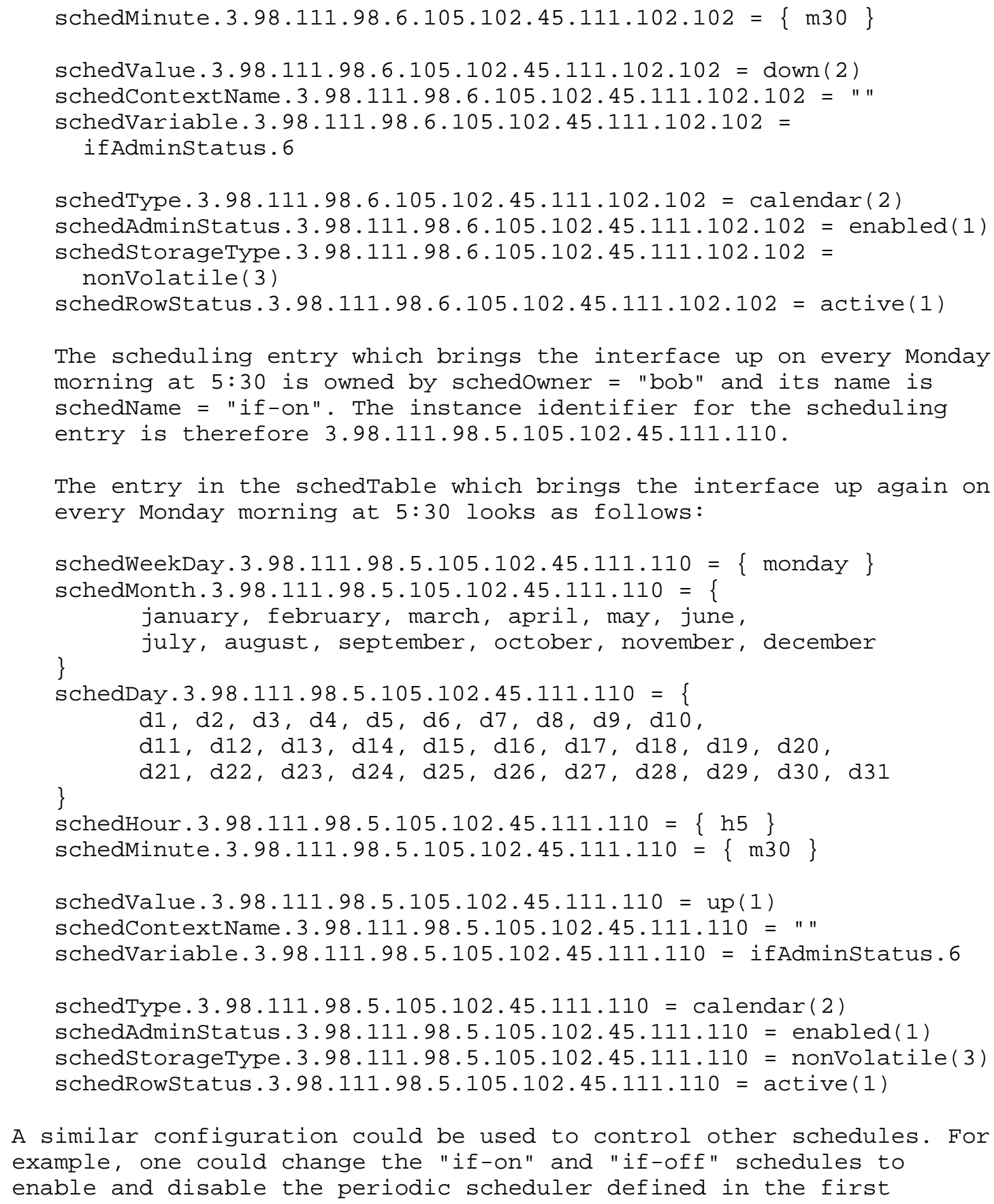

A similar configuration could be used to control other schedules. For example, one could change the "if-on" and "if-off" schedules to enable and disable the periodic scheduler defined in the first example. 


\section{Security Considerations}

Scheduled SNMP set operations must use the security credentials that were present when the corresponding row in the scheduling entry was created. An implementation must therefore record and maintain the credentials for every scheduling entry.

An implementation must ensure that access control rules are applied when doing the set operation. This is accomplished by calling the isAccessAllowed abstract service interface defined in RFC 2271 [1]:

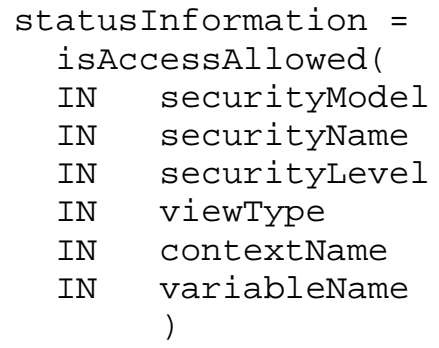

The securityModel, securityName and securitylevel parameters are set to the values that were recorded when the scheduling entry was created. The viewType parameter must select the write view and the contextName and variableName parameters are taken from the schedContextName and schedVariableName values of the scheduling entry.

This MIB limits scheduled actions to objects in the local MIB. This avoids security problems with the delegation of access rights. However, it might be possible for a user of this MIB to own some schedules that might trigger far in the future. This can cause security risks if the security administrator did not properly update the access control lists when a user is withdrawn from an SNMP engine. Therefore, entries in the schedTable sHould be cleaned up whenever a user is removed from an SNMP engine.

To facilitate the provisioning of access control by a security administrator using the View-Based Access Control Model (VACM) defined in RFC 2275 [15] for tables in which multiple users may need to independently create or modify entries, the initial index is used as an "owner index". Such an initial index has a syntax of SnmpAdminstring, and can thus be trivially mapped to a securityName or groupName as defined in VACM, in accordance with a security policy.

All entries in related tables belonging to a particular user will have the same value for this initial index. For a given user's entries in a particular table, the object identifiers for the 
information in these entries will have the same subidentifiers (except for the "column" subidentifier) up to the end of the encoded owner index. To configure VACM to permit access to this portion of the table, one would create vacmViewTreeFamilyTable entries with the value of vacmViewTreeFamilysubtree including the owner index portion, and vacmViewTreeFamilyMask "wildcarding" the column subidentifier. More elaborate configurations are possible.

\section{Intellectual Property}

The IETF takes no position regarding the validity or scope of any intellectual property or other rights that might be claimed to pertain to the implementation or use of the technology described in this document or the extent to which any license under such rights might or might not be available; neither does it represent that it has made any effort to identify any such rights. Information on the IETF's procedures with respect to rights in standards-track and standards-related documentation can be found in BCP-11. Copies of claims of rights made available for publication and any assurances of licenses to be made available, or the result of an attempt made to obtain a general license or permission for the use of such proprietary rights by implementors or users of this specification can be obtained from the IETF Secretariat.

The IETF invites any interested party to bring to its attention any copyrights, patents or patent applications, or other proprietary rights which may cover technology that may be required to practice this standard. Please address the information to the IETF Executive Director.

8. Acknowledgments

This document was produced by the IETF Distributed Management (DISMAN) working group.

9. References

[1] Harrington, D., Presuhn, R. and B. Wijnen, "An Architecture for Describing SNMP Management Frameworks", RFC 2271, January 1998.

[2] Rose, M. and K. McCloghrie, "Structure and Identification of Management Information for TCP/IP-based Internets", STD 16, RFC 1155, May 1990 .

[3] Rose, M. and K. McCloghrie, "Concise MIB Definitions", STD 16 , RFC 1212, March 1991. 
[4] Rose, M., "A Convention for Defining Traps for use with the SNMP", RFC 1215, March 1991.

[5] McCloghrie, K., Perkins, D., Schoenwaelder, J., Case, J., Rose, M. and S. Waldbusser, "Structure of Management Information Version 2 (SMIV2)", STD 58, RFC 2578, April 1999.

[6] McCloghrie, K., Perkins, D., Schoenwaelder, J., Case, J., Rose, M. and S. Waldbusser, "Textual Conventions for SMIv2", STD 58, RFC 2579, April 1999.

[7] McCloghrie, K., Perkins, D., Schoenwaelder, J., Case, J., Rose, M. and S. Waldbusser, "Conformance Statements for SMIv2", STD 58, RFC 2580, April 1999.

[8] Case, J., Fedor, M., Schoffstall, M. and J. Davin, "Simple Network Management Protocol", STD 15, RFC 1157, May 1990.

[9] Case, J., McCloghrie, K., Rose, M. and S. Waldbusser, "Introduction to Community-based SNMPv2", RFC 1901, January 1996.

[10] Case, J., McCloghrie, K., Rose, M. and S. Waldbusser, "Transport Mappings for Version 2 of the Simple Network Management Protocol (SNMPV2)", RFC 1906, January 1996.

[11] Case, J., Harrington D., Presuhn R. and B. Wijnen, "Message Processing and Dispatching for the Simple Network Management Protocol (SNMP) ", RFC 2272, January 1998.

[12] Blumenthal, U. and B. Wijnen, "User-based Security Model (USM) for version 3 of the Simple Network Management Protocol (SNMPv3)", RFC 2274, January 1998 .

[13] Case, J., McCloghrie, K., Rose, M. and S. Waldbusser, "Protocol Operations for Version 2 of the Simple Network Management Protocol (SNMPV2)", January 1996.

[14] Levi, D., Meyer, P. and B. Stewart, "SNMPv3 Applications", RFC 2273, January 1998

[15] Wijnen, B., Presuhn, R. and K. McCloghrie, "View-based Access Control Model (VACM) for the Simple Network Management Protocol (SNMP) ", RFC 2275, January 1998.

[16] Hovey, R. and S. Bradner, "The Organizations Involved in the IETF Standards Process", BCP 11, RFC 2028, October 1996. 
[17] Levi, D. and J. Schoenwaelder, "Definitions of Managed Objects for the Delegation of Management Scripts", RFC 2592, May 1999.

[18] McCloghrie, K. and F. Kastenholz, "The Interfaces Group MIB using SMIv2", RFC 2233, November 1997.

[19] Bradner, S., "Key words for use in RFCs to Indicate Requirement Levels", BCP 14, RFC 2119, March 1997.

10. Editors' Addresses

David B. Levi

Nortel Networks

4401 Great America Parkway

Santa Clara, CA 95052-8185

U.S.A.

Phone: +1 4236860432

EMail: dlevianortelnetworks.com

Juergen Schoenwaelder

TU Braunschweig

Bueltenweg $74 / 75$

38106 Braunschweig

Germany

Phone: +49531 391-3283

EMail: schoenwlibr.cs.tu-bs.de 
11. Full Copyright statement

Copyright (C) The Internet Society (1999). All Rights Reserved.

This document and translations of it may be copied and furnished to others, and derivative works that comment on or otherwise explain it or assist in its implementation may be prepared, copied, published and distributed, in whole or in part, without restriction of any kind, provided that the above copyright notice and this paragraph are included on all such copies and derivative works. However, this document itself may not be modified in any way, such as by removing the copyright notice or references to the Internet society or other Internet organizations, except as needed for the purpose of developing Internet standards in which case the procedures for copyrights defined in the Internet Standards process must be followed, or as required to translate it into languages other than English.

The limited permissions granted above are perpetual and will not be revoked by the Internet society or its successors or assigns.

This document and the information contained herein is provided on an "AS IS" basis and THE INTERNET SOCIETY AND THE INTERNET ENGINEERING TASK FORCE DISCLAIMS ALL WARRANTIES, EXPRESS OR IMPLIED, INCLUDING BUT NOT LIMITED TO ANY WARRANTY THAT THE USE OF THE INFORMATION HEREIN WILL NOT INFRINGE ANY RIGHTS OR ANY IMPLIED WARRANTIES OF MERCHANTABILITY OR FITNESS FOR A PARTICULAR PURPOSE.

Acknowledgement

Funding for the RFC Editor function is currently provided by the Internet society. 